\title{
Lattice gas simulations of dynamical geometry in two dimensions
}

\author{
Anna Klales, ${ }^{1}$ Donato Cianci, ${ }^{1}$ Zachary Needell, ${ }^{1}$ David A. Meyer, ${ }^{2}$ and Peter J. Love ${ }^{1,3}$ \\ ${ }^{1}$ Department of Physics, Haverford College, 370 Lancaster Avenue, Haverford, Pennsylvania 19041, USA \\ ${ }^{2}$ Department of Mathematics, UCSD, La Jolla, California 92093, USA \\ ${ }^{3}$ Institute for Quantum Information, California Institute of Technology, Pasadena, California 91125, USA \\ (Received 11 February 2010; revised manuscript received 30 June 2010; published 12 October 2010)
}

\begin{abstract}
We present a hydrodynamic lattice gas model for two-dimensional flows on curved surfaces with dynamical geometry. This model is an extension to two dimensions of the dynamical geometry lattice gas model previously studied in one dimension. We expand upon a variation of the two-dimensional flat space FrischHasslacher-Pomeau (FHP) model created by Frisch et al. [Phys. Rev. Lett. 56, 1505 (1986)] and independently by Wolfram, and modified by Boghosian et al. [Philos. Trans. R. Soc. London, Ser. A 360, 333 (2002)]. We define a hydrodynamic lattice gas model on an arbitrary triangulation whose flat space limit is the FHP model. Rules that change the geometry are constructed using the Pachner moves, which alter the triangulation but not the topology. We present results on the growth of the number of triangles as a function of time. Simulations show that the number of triangles grows with time as $t^{1 / 3}$, in agreement with a mean-field prediction. We also present preliminary results on the distribution of curvature for a typical triangulation in these simulations.
\end{abstract}

DOI: 10.1103/PhysRevE.82.046705

PACS number(s): 02.70.Ns, 05.20.Jj, 05.70.Ln

\section{INTRODUCTION}

Lattice gas automata (LGA) models for fluids date from the 1960s, when Kadanoff and Swift [1] and Hardy et al. [2] introduced the first such models. Both of these models use a two-dimensional (2D) Cartesian lattice and are anisotropic. Since simple fluids are isotropic, these models are not capable of reproducing hydrodynamics. This problem was solved in 1986 when Frisch et al. [3], and independently Wolfram [4], introduced an isotropic model [the FrischHasslacher-Pomeau (FHP) model] for the Navier-Stokes equations using a triangular lattice. These models were subsequently extended to three dimensions and generalized to model complex flows [5]. These models led directly to the creation of lattice-Boltzmann models which are now widely used for simple and complex fluid flow simulations [6].

All LGA methods evolve by propagation and collision of particles on a lattice. During propagation, particles move from site to site on the lattice, while during collision the particles rearrange themselves among the vectors at each lattice site. It is important to note that the rules that govern these models are not meant to replicate the physical world on a small scale; the Navier-Stokes equations emerge from the FHP rules on the macroscopic scale for large lattice sizes and spatial or ensemble averaging. The microscopic rules are only required to conserve total momentum, particle number, and energy. Additionally, the lattice must be sufficiently symmetric to yield an isotropic pressure tensor.

Many 2D situations of physical interest use a Euclidean plane as the underlying geometry, hence "lattice" gases, in which the model is constructed on a translation-invariant discretization of Euclidean space. However, situations exist where fluids flow on curved surfaces, such as atmospheric flow [7], flows in curved soap films (for example, the experiments of Seychelles et al. [8]), or surface flows in interfaces embedded in fluid mixtures (for example, in amphiphilic fluids [9]). In these cases a discretization of a sphere or other surface in which the geometry is non-Euclidean may be more appropriate. In such geometries, the presence of curvature means that the angles of a triangle need not sum to $\pi$. In the discrete case we may specialize to simplicial complexes made up of equilateral triangles, as any 2D surface may be discretized in this way [10]. In this case the geometry is defined locally by the number of triangles meeting at each grid point. If six triangles meet, the geometry is locally flat. If fewer than six triangles meet, the geometry has positive local curvature concentrated at that vertex. If more than six triangles meet, the geometry has negative local curvature. If the properties of the triangulation, including the local curvature, are allowed to change we call the geometry dynamical.

There are many situations in physics in which geometry takes on a dynamical role. Perhaps the most fundamental is in Einstein's general theory of relativity, in which the idea of motion along geodesics in a Riemannian manifold supervenes Newtonian ideas of acceleration due to forces [11]. In the Regge treatment of general relativity [12] and the causal dynamical triangulations approach to quantum gravity $[13,14]$ these Riemannian manifolds are replaced with simplicial complexes.

The statistical mechanics and growth dynamics of random surfaces have been much studied for both onedimensional interfaces [15] and 2D surfaces [16-19]. In spite of their origins in very different physical systems, the common language of discretized surfaces can be informative. For example, the crumpling transition of membranes [19] also occurs in Euclidean approaches to simplicial quantum gravity [13].

In soft-matter physics, the presence of interfaces in fluid mixtures can give rise to mesophases with complex rheology. Variations in surface tension on a fluid interface can drive Marangoni flows which can in turn drive interfacial dynamics [20]. These instabilities have been observed in lattice gas simulations of amphiphilic fluids [21]. The equations which govern surface flow have been known for some time [22,23]; however, numerical simulation methods which can treat fluid flow on curved surfaces with dynamical geometry clearly present a challenge. 
Early modeling of complex fluid phases used Isingmodel-inspired ideas to develop lattice models that represent the static mesophases in equilibrium. For example, Widom's model of microemulsions used an Ising model to represent interactions among oil, water, and amphiphile phases [24]. Similarly, the equilibrium models of random surfaces referred to above can be described in terms of an Ising-type interaction between the surface normals of triangles. The lattice gas model of microemulsions defined in [25] and investigated in $[21,26-32]$ can be understood as a dynamical generalization of Widom's model. The model presented here is in the spirit of a dynamical generalization of the models of $[16,17]$ in which the surface now carries a vector field (the velocity defined by the lattice gas model) and where the dynamics of the surface are coupled to this field.

In this paper we present a hydrodynamic lattice gas model for two-dimensional flows on curved surfaces with dynamical geometry. We extend a variation of the FHP model to arbitrary equilateral triangulations. We allow the geometry so defined to become dynamical by applying the Pachner moves contingent on the particle content. Time reversibility is used as a guide to restrict the rule space, as in the one-dimensional version of this model [33-35]. We present a mean-field prediction and simulation results for the growth of the lattice as a function of time, and give preliminary results on the distribution of curvature on the triangulations generated by these simulations. We close the paper with some conclusions and directions for future work.

\section{FHP MODEL ON AN ARBITRARY SURFACE}

We first distinguish extrinsic geometry from intrinsic geometry. When specifying a triangulation, one can use an extrinsic definition of the geometry, or an intrinsic definition. An extrinsic definition describes the triangulation by relating it to an ambient or embedding space. For example, a tetrahedron can be defined extrinsically by giving the Cartesian coordinates of its vertices in three-dimensional Euclidean space.

Geometry can also be defined intrinsically, without reference to embedding in some higher-dimensional space. For example, we can define a tetrahedron intrinsically as follows. First, we specify that a triangle is defined by three points equidistant from each other. Then, we specify that we have four triangles, and that each triangle shares exactly one edge with every other triangle. This is illustrated in Fig. 1. We have defined a tetrahedron intrinsically. There was no reference to any coordinate system, only reference to parts of the triangulation itself.

For a lattice gas model defined on an arbitrary triangulation the flux of particles defines a velocity field. A velocity vector on a triangulation lives in the tangent space to the triangulation at that point. In general, transport of tangent vectors on manifolds requires a description of the relationship between tangent spaces at different points on the manifold. For example, when computing a covariant derivative on a Riemannian manifold one must consider the variation of coordinate basis vectors with positions on the manifold. The components of the derivatives with respect to the coordinates

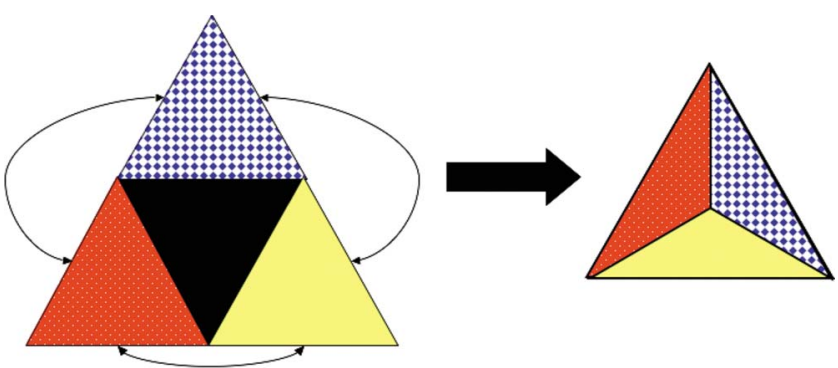

FIG. 1. (Color online) A triangle whose neighbors are neighbors belongs to a tetrahedron. This is an intrinsic definition of a tetrahedron. On the left there are three triangles sharing one edge each with the black triangle. They are the black triangle's neighbors. If the blue (top), red (bottom left), and yellow (bottom right) triangles in the left figure are also neighbors as designated by the arrows above, the three triangles fold into a tetrahedron (right).

of the basis vectors are the Christoffel symbols, which specify the connection on the manifold. These quantities are intrinsic: they may be computed from the metric without reference to any higher-dimensional embedding space.

In the FHP lattice gas automata the particles move on a triangular lattice. At each lattice site there are six lattice vectors. Each vector can be occupied by at most one particlethe model has an exclusion principle. The vector which a particle occupies defines its velocity. In Fig. 2, for example, the site is occupied by a single particle moving to the right. The state of a particular site is given by its particle content. Each vector at each site can have two states: occupied or unoccupied. This gives a total of $2^{6}=64$ states per site.

We now generalize the FHP model to arbitrary equilateral triangulations. It is known that any manifold can be approximated arbitrarily closely by a tiling of equilateral triangles [10]. To generalize from a two-dimensional Euclidean lattice to an arbitrary triangulation, we would like to regard our sites as triangles rather than as single points. We therefore enclose the site in a triangle and move the vectors to the edges of the triangles, as demonstrated in Fig. 2. This "inflated" site is equivalent to the point site used by Frisch $e t$ al. This allows us to triangulate any surface and regard each face as an inflated FHP site. In the special case of flat space the vectors in the array of inflated sites (Fig. 2) create a tiling

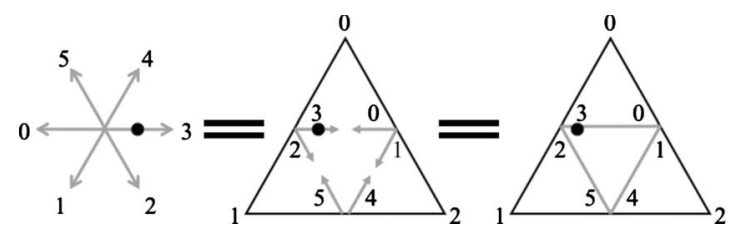

FIG. 2. An FHP lattice site has six possible velocities labeled $0-5$, each of which represents the velocity of a particle. Each vector can hold at most one particle, so that each site has $2^{6}=64$ states The traditional representation of a site in the FHP model is the star shown on the left. By moving the vectors to the edges of a triangle, as shown in the center picture, we convert the site from a single point to the face of a triangle. These two sites are equivalent. If we remove the arrow heads from the vectors, we produce the notationally convenient right-hand figure. We refer to the conversion between the star and the triangle as inflation. 


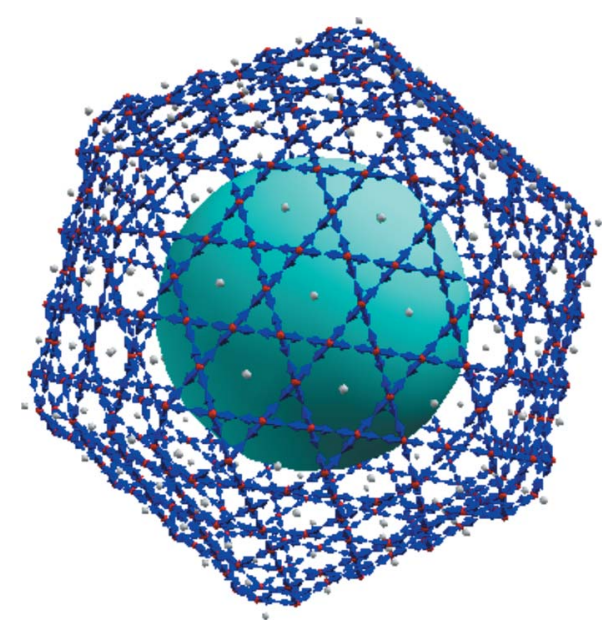

FIG. 3. (Color online) A kagome lattice on an icosahedron. The nodes of the triangulation are depicted as unconnected spheres. This image was generated with visual python [36].

of Stars of David, along the lines of which the particles can move. This lattice is known as the kagome lattice. Figures 3 and 4 show triangulations of an icosahedron and a cylinder. These images were generated with visual python [36].

This modification of the FHP model was originally proposed by Boghosian et al. [37]. Those authors proposed collisions on the edges of the triangles, where four vectors meet, and introduced the possibility of having a rest particle of mass 2 in the model for a total of 5 bits per state. This model was analyzed by a grouping of triplets of triangle edges sites followed by a Chapman-Enskog expansion which yielded isotropic fluid equations. However, collisions at the vertices of the kagome lattice result in an extra component of conserved momentum. No momentum is transferred between separate lines of the lattice, so momentum is conserved in three directions in two-dimensional space. This leads to unphysical flows. We therefore redefine the model with collisions occurring on the faces, rather than the edges, of the triangle. It should be noted that the Chapman-Enskog analysis presented in [37] remains valid for a model, such as the one we present here, in which momentum is exchanged by collisions among all lattice directions.

The rules used for fixed geometry in the variant of the FHP model we study are shown graphically in Figs. 7(a) and 7(b). If two particles enter a site with opposite velocities, as

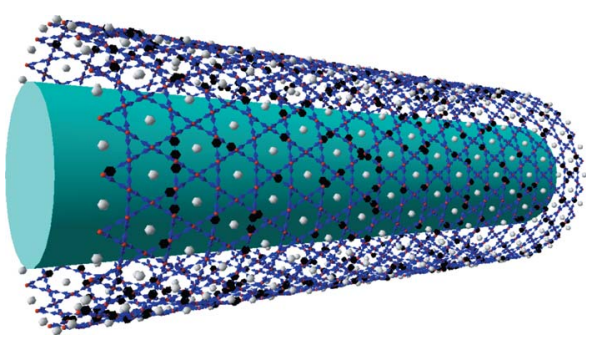

FIG. 4. (Color online) A kagome lattice on a cylinder. The nodes of the triangulation are depicted as unconnected spheres. Particles are allowed to move along the lines of the kagome lattice and are shown in black. This image was generated with visual python [36].
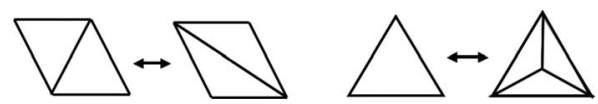

FIG. 5. Pachner moves. A sequence of Pachner moves can connect any pair of triangulations of a manifold, but cannot change the topology. The two-to-two move (left) changes the orientation of two triangles as shown above, and the one-to-three move (right) replaces one triangle with three, creating a tetrahedron, or vice versa.

in Fig. 7(a), they flip to either of the other lines of the lattice with equal probability. If three particles enter a site such that their total momentum sums to zero-in other words, there is a particle occupying every other vector-the particles switch from the occupied vectors to the unoccupied vectors, shown in Fig. 7(b). This three-body collision breaks the separate conservation of momentum along each line of the lattice. If particles enter in any other configuration, they are simply allowed to propagate as usual to the next site along their geodesic [3]. We now describe the rules which couple the particles to the triangulation and allow the geometry to become dynamical.

We employ the Pachner moves to change the triangulation. A sequence of Pachner moves cannot change the topology of a manifold, but it can take the manifold from one triangulation to another: a torus can morph into another toroidal geometry such as a coffee mug, but it cannot morph into a sphere [10]. The state of the system with static and flat geometry is specified by the particle content of the sites alone; the state of the system with dynamical geometry is specified by both the particle content and the geometry of the triangulation.

There are two Pachner moves for two-dimensional triangulations: a two-to-two move, where the number of triangles is unchanged, and a one-to-three or three-to-one move that increases or decreases the number of triangles by two. We call the three-to-one and the one-to-three moves deletion and addition, respectively, because they add or subtract a tetrahedron from the surface.

The effect of the Pachner moves on the intrinsic geometry of the model is well defined. However, the effect on the extrinsic geometry requires some thought. Consider embedding or immersing [38] an equilateral triangulation of a surface in three dimensions. The two-to-two Pachner move (Fig. 5 , left) is not isometrically embeddable or immersible in general. If two triangles are removed, turned, and replaced in the triangulation, they will not fit unless the dihedral angle between the original pair of triangles was that of the tetrahedron. This is unsurprising since two-dimensional manifolds are not generically embeddable or immersible in three dimensions [39]. However, the one-to-three move (Fig. 5, right) is generically immersible, although it is not generically embeddable because it may cause self-intersection of the surface.

To couple the flow to the geometry we must specify how the application of a particular Pachner move is triggered by the particle content. The rules for fixed geometry involve particles on a single triangle. The locality of a rule which changes the geometry is determined by the locality of the Pachner moves. The one-to-three, three-to-one, and two-to- 


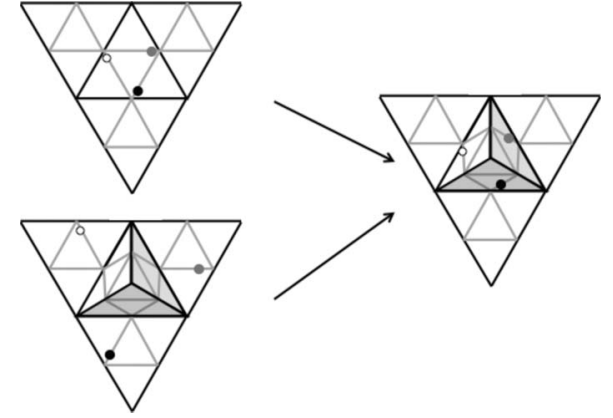

FIG. 6. If new geometry is not created empty one state may have two preimages, a problem illustrated in this figure. Both figures on the left would produce the figure on the right if they were allowed to time evolve. The particles on the triangle in the upper left-hand corner are therefore required to propagate off before the new geometry is created. This allows rules with dynamical geometry in which every state has a unique preimage, and which are therefore invertible.

two moves are triggered by the state of one, three, or two triangles, respectively.

As in one dimension, the constraint of time reversibility is used in order to restrict the set of rules considered [33]. We first recall the distinction between invertibility and reversibility. A rule is invertible if every state has a unique preimagegiven the state (particle content plus geometry) one may reconstruct the whole unique history leading to that state for an invertible rule. For a reversible lattice gas rule, the history of a given state may be generated by an inverse rule which can be interpreted as propagation and the same collision rules that generate the forward time evolution. One must recall that the inverse of the product of collision and propagation, $C P$, is $P^{-1} C^{-1}$; and so to construct the inverse of a rule one must reverse the order in which collision and propagation are applied. The restriction of time reversibility is satisfied if we create new geometry after the particles have propagated from the triangle. That is, we do not create new geometry that contains particles. If we created new geometry with particle content, the resulting state may have two preimages: one preimage in which the geometry is about to be created and one preimage in which particles are about to advect onto existing geometry. This problem is illustrated in Fig. 6.

We first consider the one-to-three move in which new geometry is created. In the propagation phase of the code triangles which have just undergone the three-body collision shown in Fig. 7(b) are flagged as sites where new geometry will be added. The three particles which have just collided will propagate off this triangle, and new particles will propagate onto it. The propagation step is performed, and then the next round of collisions and geometry update occur. Note that we allow "spectator" particles to propagate onto the existing geometry before the addition occurs. Furthermore, if these spectator particles form preimages of a one- or twobody collision as defined in Fig. 7(a) or Fig. 7(b) that collision takes place before the addition of geometry. The presence of these particles means that the "new geometry created empty" rule is being used as a guide to the construction of the model rather than a prescription.
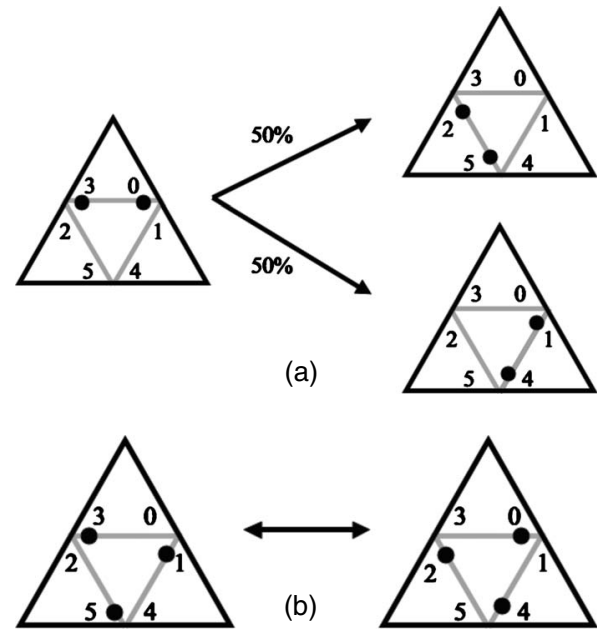

FIG. 7. (a) A two-particle collision at a site. The particles switch with equal probability to one of the other two directions of the lattice. This rule applies to any two particles entering a site with opposite velocities. (b) A three-particle collision at a site. The particles switch to the unoccupied vectors of the site. This move breaks the separate conservation of momentum along each direction of the lattice.

The rule for deletion is illustrated in Fig. 8(b). When three particles propagate off a tetrahedron in the same manner, the tetrahedron is deleted [see Fig. 8(b)]. The tetrahedra which have the correct particle occupancy to be deleted are identified before the propagation step. The particles then propagate off the tetrahedron (and new particles propagate onto its boundary), and the tetrahedron is deleted. The particle state resulting from propagation of spectator particles onto the tetrahedron cannot be a preimage of the collisions shown in Fig. 7(a) or Fig. 7(b). The deletion of the tetrahedron can therefore occur concurrently with the subsequent collision as shown in Fig. 8(b).

We now determine rules for applying the two-to-two move. This move does not create new geometry, and so it is
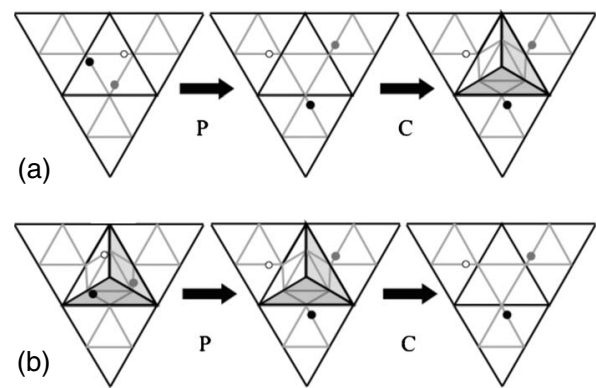

FIG. 8. (a) Creation: a triangle is replaced with a tetrahedron. This collision is triggered by a three-particle collision, where three particles enter a site on every other vector (all odd numbered or all even numbered vectors) such that the combined momentum of the three particles is zero. The particles then propagate away, and the tetrahedron is formed. (b) Deletion: three triangles forming a tetrahedron are replaced with a single triangle. This collision is triggered when three particles propagate off the tetrahedron, as shown on the left above. This also happens when the particles leave the tetrahedron on the empty vectors that point to the surrounding triangles. 


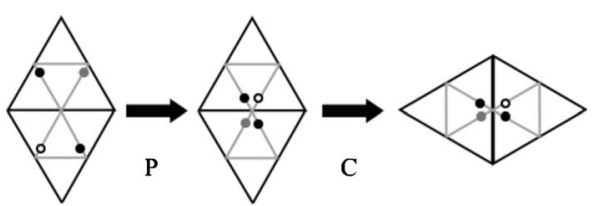

(a)

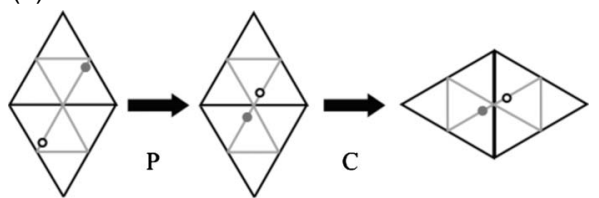

(b)

FIG. 9. The two-to-two move is triggered by two different states: (a) the four-particle state and (b) the two-particle state. The particles remain where they are during these moves.

straightforward to ensure that the rule is time reversible. This move is triggered by two different states: a four-particle state and a two-particle state, shown in Figs. 9(a) and 9(b). The pairs of triangles which are in the states shown as preimages of propagation in Figs. 9(a) and 9(b) are labeled at propagation, and the two-to-two move is applied at the subsequent collision step.

The rules including dynamical geometry are therefore modified from the stochastic FHP rules defined in Figs. 7(a) and 7 (b) by the fact that the three-body rule of Fig. 7(b) is followed by the one-to-three Pachner move shown in Fig. 8(a). The stochastic two-body rules remain unchanged. The rule set also includes the two-to-two Pachner moves shown in Figs. 9(a) and 9(b) in which the geometry changes but the particle states do not. The inclusion of the stochastic twobody rule of Fig. 7(a) and the fact that we allow spectator particles during geometry creation render the model as a whole irreversible. This could be remedied by forbidding spectators during geometry change, and the replacement of the stochastic two-body rule with a deterministic rule, requiring the addition of a rest particle, as described in [37]. Here, we avoid the use of a rest particle and retain the stochastic two-body rule and the spectator particles. An example evolution of five triangles under these rules is shown in Fig. 10.

\section{CONSERVED QUANTITIES}

Hydrodynamic lattice gases are so called because the collision and propagation rules preserve mass and momentum. Writing a Boltzmann equation for the gas and performing a Chapman-Enskog expansion about the local equilibrium distribution results in the incompressible Navier-Stokes equations for the average particle velocity. For the generalization of the FHP model to flows on arbitrary triangulations, mass is evidently still conserved; however, the role of conservation of momentum requires careful consideration.

When the model is restricted to a flat two-dimensional triangulation the FHP model is recovered, and the same arguments may be used to recover the Navier-Stokes equations. If this triangulation is embedded in three-dimensional space as shown in Fig. 4, the momentum of particles in the embedding space is not preserved when they move around the cylinder, although the geometry has no intrinsic curva-

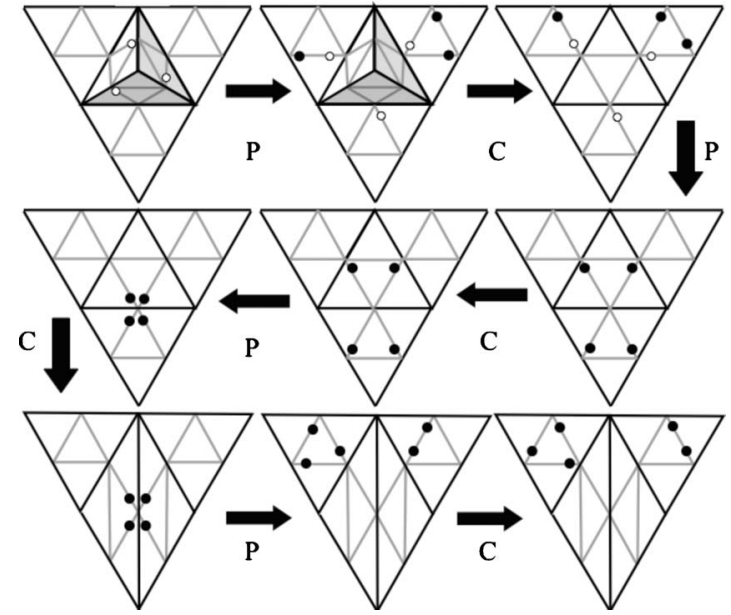

FIG. 10. An example evolution under the rules defined above. The evolution begins with a preimage of propagation, which leads to deletion of a tetrahedron. Two-body and three-body collisions occur on the neighboring triangles. No collisions occur at the subsequent step, but a two-to-two move is triggered at the following step, followed by two- and three-body collisions. Particles propagate on and off the triangles shown here during the propagation steps.

ture. This means that unrolling the cylinder by means of a coordinate transformation can recover the flat space fluid equations.

If we consider a flow on a surface such as that shown in Fig. 3 we see that in the flat regions on the faces of the icosahedron we can expect to recover the Navier-Stokes equations. Where two such regions meet the embedding space momentum is no longer conserved as the particles change directions; however, there is no intrinsic curvature at these points. A coordinate transformation may be applied to a patch extending over two faces, which recovers flat space fluid mechanics. Where three of the faces of the icosahedron meet there is a point of intrinsic curvature which will prevent any coordinate transformation from recovering flat space fluid mechanics. The point of curvature will deflect particle trajectories, and so the embedding space momentum is not conserved, but also it is not possible by a coordinate transformation to recover a two-dimensional model in which the momentum is conserved.

In what sense, therefore, may this model be described as hydrodynamic when implemented on surfaces with arbitrary intrinsic curvature and arbitrary embeddings? First, we note that the conservation properties of the flat space FHP model may be divided into conservation separately by collision and propagation. In the model defined above on an arbitrary triangulation without dynamical geometry momentum defined locally at a site is still conserved by collisions.

For a curved surface embedded in three-dimensional space the momentum of a particle may change under propagation for one of two reasons. Either the propagation may cause it to deviate from a straight line in embedding space, as in the case of particles moving on the cylinder shown in Fig. 4, or intrinsic curvature of the surface may cause the path of the particle to deviate from a straight line. However, in both of these cases propagation moves the particle along a 
geodesic of the triangulation. In the case that the triangulation is a good approximation to a smooth manifold propagation moves the particles along paths which approximate solutions to the geodesic equation of motion.

If we interpret the model defined on an embedded surface as a flow on, for example, an embedded fluid interface, the deviation of the particles from straight lines in embedding space must be interpreted as the result of a force holding the particles on the surface. Restricting the flow to the surface without explicitly including these forces in the model amounts to treating the restoring forces normal to the interface as constraint forces. This approach is justified provided that these restoring forces are much larger than any other force in the model-equivalently, that there is a large time scale separation between the short time scales on which particles displaced from the interface are returned to it and the longer time scales on which particles are transported within the interface. However, such a separation of forces and time scales is necessary for a well-defined interface to exist.

Naturally, given constraint forces which restrict the flow to a curved two-dimensional manifold, hydrodynamics remains well defined. The Navier-Stokes equations on the sphere are well known, and the effects of curvature on any manifold may be included by the usual prescription of replacing spatial derivatives with covariant derivatives. The elucidation of a lattice gas model for hydrodynamics on an arbitrary manifold is beyond the scope of the present paper, in which we consider the dynamics of the geometry in a quiescent fluid. However, in related work we show that the usual Chapman-Enskog procedure to obtain hydrodynamic equations from lattice gas and lattice-Boltzmann methods may be extended to arbitrary manifolds [40].

\section{IMPLEMENTATION}

Implementation of the rules defined in the previous section presents several challenges. In this section, we describe some of the details of our implementation which allow the model to be efficiently simulated without reference to embedding space coordinates.

The implementation of our model contains both intrinsic and extrinsic geometry information. The extrinsic (embedding space) information is the set of vertex coordinates, velocity vectors, and particle coordinates of our twodimensional simulation in three-dimensional space. This is used to produce visualizations of the model, such as those shown in Figs. 3 and 4. It is possible to imagine situations in which the extrinsic information is coupled to the intrinsic model dynamics. For example, a membrane embedded in a bulk fluid will have dynamics driven in part by the embedding space fluid dynamics. We only consider model dynamics defined intrinsically. In particular this means that the dynamics remains perfectly well defined even if the triangulation is no longer isometrically embeddable in threedimensional Euclidean space. We do include the possibility that moves which would be allowed by the intrinsic dynamics are forbidden conditioned on the embedding; however, for all simulations described in this paper these constraints were inactive.
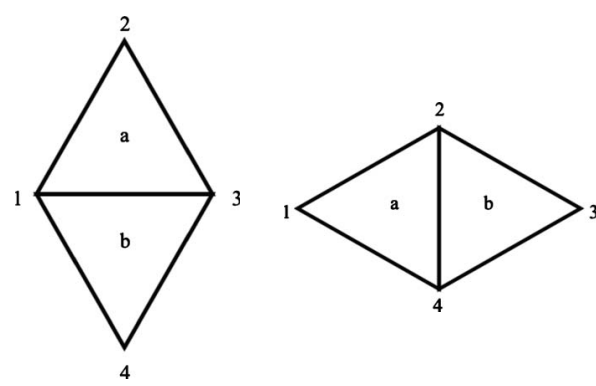

FIG. 11. The two-to-two Pachner move. The pair of triangles is rotated in the lattice, so that vertex 1 goes to vertex 2, vertex 2 goes to vertex 3 , vertex 3 goes to vertex 4 , and vertex 4 goes to vertex 1 . The relationship between triangles $\mathbf{a}$ and $\mathbf{b}$ stays the same; only the connectivity between each triangle and the surrounding triangles is redefined, along with the vertices of each triangle.

The collision rules are defined locally and must conserve local fluxes of mass, momentum, and energy of the particles. We wish to apply the same collision rule on every triangle expressed in terms of the vector labels. In general, translation of a triangle from one location on the triangulation to another will induce a transformation of the vector labels. A reflection of the vector labels through one of the symmetry axes of the triangle will change the definition of momentum between one triangle and another. Because of this we restrict to labelings in which the transformation relating the vector labelings of any two triangles is one of the three proper rotations of the labeled triangle shown in Fig. 2.

The propagation rule moves particles from one triangle to another. This operation depends on the transformation of the labeling of vectors on going from one triangle to another. For each triangle each of the six vectors carries two pieces of connectivity information which define this transformation. First each vector carries a triangle label which gives the triangle reached by propagation along that vector. Second each vector carries a vector label which determines the vector the particle arrives at after propagation. Because the vector labelings of any two sites are related by one of the three rotations, the labeling of two adjacent triangles is determined by the image of any one of the vectors. Hence, the connectivity information redundantly determines the geometry.

\section{A. Implementing the two-to-two Pachner move}

The two-to-two Pachner move changes the orientation of two neighboring triangles but not the number of triangles. After a collision applying such a move it is necessary to change the connectivity information of the surrounding triangles. The move is shown in Fig. 11, where triangles a and b form a rhombus. During the two-to-two move, the four vertices of the rhombus undergo a cyclic permutation as the rhombus rotates. The connectivity between the two triangles involved in the move and the surrounding triangles must be updated, and the positions of the vertices in embedding space will change unless the dihedral angle between $\mathbf{a}$ and $\mathbf{b}$ is that of the tetrahedron. The connectivity between triangles a and b does not change.

Triangle pairs which are candidates for the two-to-two move are identified before the propagation phase. Triangles 


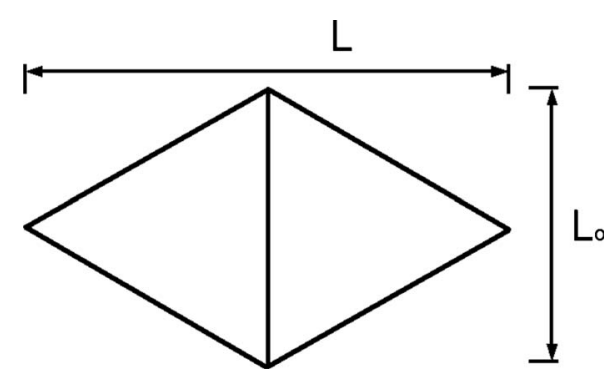

FIG. 12. If the new edge $L$ is more than a fraction $x$ different from $L_{0}$, the two-to-two move is prevented.

in the appropriate states, for example, the pair of triangles in the leftmost panel of Figs. 9(a) and 9(b), are identified. At the subsequent collision the move is performed; connectivity is redefined with the surrounding triangles, and the vertices of the triangles are updated.

As noted above, unless the dihedral angle of the two original triangles is that of the tetrahedron, applying the twoto-two move will result in a triangulation which is not embeddable or immersible in three-dimensional Euclidean space. A control exists in the code which prevents the change in the embedded length of edges from deviating from the equilateral value by more than a specified fraction. If $L_{0}$ is significantly different from $L$ as shown in Fig. 12, i.e., the new edge is significantly different from the new edge, we do not allow the two-to-two move to be performed. Given a fraction $x \quad(0<x \leq 1)$, we determine whether or not the change will be performed via the restriction

$$
(1-x) L_{0}<|L|<(1+x) L_{0} .
$$

What values of $x$ are relevant? For an initially flat triangulation, $L=\sqrt{3} L_{0}$; and for a single tetrahedron added to an initially flat triangulation, $L=\sqrt{2} L_{0}$. For triangles which meet with the dihedral angle of the icosahedron, $L=\phi L_{0} / 2$, where $\phi$ is the golden ratio. Hence, for $x<(\sqrt{2}-1)$ with an initially flat triangulation no two-to-two moves will be performed. For $x<(\sqrt{3}-1)$ positive curvature added to an initially flat triangulation by a one-to-three move is frozen in place at the new vertex, as no two-to-two moves may be applied involving any face of the tetrahedron. For $x<(\phi / 2-1)$ no two-totwo moves may be applied to an icosahedron.

The effect of these moves is therefore to increase the edge lengths of the triangles according to the Euclidean metric in three-dimensional embedding space. One may imagine the triangulation inflated by a scale factor and embedded isometrically in a higher-dimensional space in such a way that the three-dimensional embedding is a projection of this higher-dimensional embedding. In this case the restriction specified by $x$ is to triangulations whose projections into three-dimensional Euclidean space are almost isometric. Alternatively, one may regard the three-dimensional embedding space itself as no longer Euclidean. In this case, $x$ represents a bound on the deviation of the metric of the threedimensional embedding space from Euclidean. Note that because $x$ specifies a ratio between new and old embedded lengths, this constraint allows triangle edge lengths to grow repeatedly by a succession of geometry-changing moves.
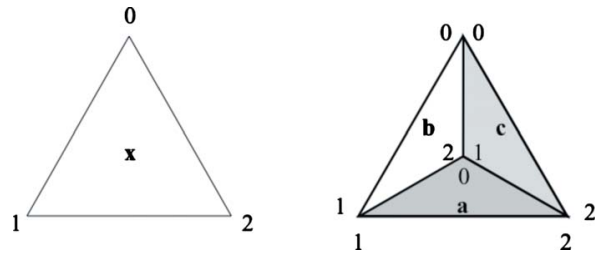

FIG. 13. (Color online) The one-to-three Pachner move (creation). The triangles are labeled $\mathbf{x}, \mathbf{a}, \mathbf{b}$, and $\mathbf{c}$, and the vertices are labeled by 0,1 , and 2 . ( $\mathbf{m}^{n}$ refers to triangle $m$ 's $n$th vertex). When triangle $\mathbf{x}$ is triggered for addition, copies of triangle $\mathbf{x}$ are rotated out of the plane of the paper along each edge. For example, to produce triangle $\mathbf{b}$ triangle $\mathbf{x}$ is copied and rotated along the edge joined by the vertices $\mathbf{x}^{1}$ and $\mathbf{x}^{0}$, matching vertex $\mathbf{x}^{2}$ with the apex. Acting similarly for the other two sides, triangle $\mathbf{x}$ on the left transitions to the tetrahedron on the right. Deletion is the inverse of this process, and intersections of common vertices and triangle neighbors are used to identify the relevant vertices. The apex is identified as the intersection of the vertices of triangles $\mathbf{a}, \mathbf{b}$, and $\mathbf{c}$, since the apex is the only vertex shared by all three triangles.

One could also implement a constraint which would bound all embedded edge lengths above by an additive constant. We leave the investigation of this possibility for future work.

\section{B. Implementing the one-to-three Pachner move: Addition}

Triangles triggered for addition are marked before propagation and undergo changes in geometry after the subsequent collision. First, the embedding space coordinates of the apex of the new tetrahedron are determined. The three triangles of the new tetrahedron each have two of the vertices of the triggered triangle, and the third vertex is the apex. The move may be regarded as making three copies of the original triangle and "rotating" each triangle along a different edge, so that its free vertex becomes the apex. In Fig. 13, triangle a has been rotated along the 1-2 edge, triangle $\mathbf{b}$ has been rotated along the $0-1$ edge, and triangle $\mathbf{c}$ has been rotated along the 0-2 edge. One of the three new triangles replaces the original. The connectivity of the new tetrahedron is set to be that shown in Fig. 14 where $\mathbf{a}$ is the original triangle and $\mathbf{b}$ and $\mathbf{c}$ are the two added triangles.

The curvature at any triangle vertex is equal to six minus the number of triangles meeting at that vertex. If the one-tothree Pachner move is implemented without restriction, vertices of the triangulation with arbitrarily large negative curvature may form. This is because the one-to-three move adds a new vertex with positive curvature and increases the number of triangles meeting at each of the original three vertices of the triangle by one. In order to allow simulations in which the curvature is bounded between $\pm c$, we forbid addition of tetrahedra on a triangle with any vertex with curvature $c$. Bounding the curvature to be \pm 1 from the original triangulation is equivalent to preventing new tetrahedra from forming on existing tetrahedra.

\section{Implementing the three-to-one Pachner move: Deletion}

The deletion rule depends on the state of three triangles in a tetrahedral configuration. Tetrahedra are identified as sets 


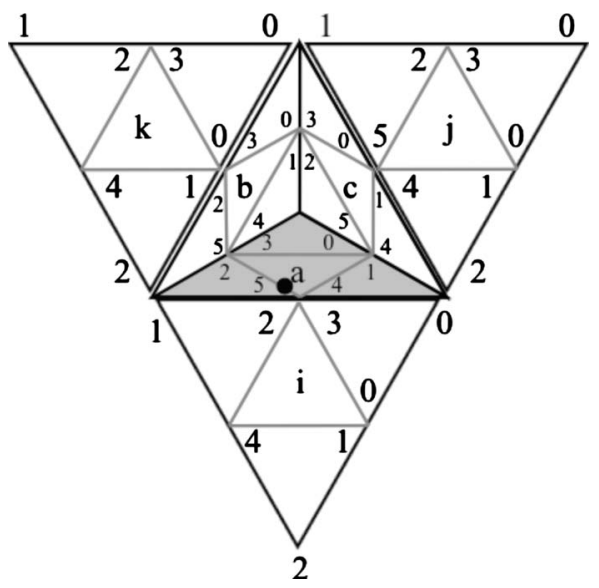

FIG. 14. When a tetrahedron undergoes deletion, the connectivity must be redefined. Here, the tetrahedron is being replaced by triangle a.

of triangles whose neighbors are neighbors using the intrinsic information-this test uniquely specifies a tetrahedron (see Fig. 1). Once tetrahedra have been identified, they are checked to see if their particle content makes them a preimage of deletion.

Deletion presents a computational issue, in that triangles must be removed from the list in which they are stored. This would change the indices of all triangles, requiring a relabeling of the whole triangulation, a computationally demanding process. It is more efficient to disregard the triangles that have been deleted and place them on a dummy list. These obsolete triangles are ignored whenever the state is updated, and hence relabeling is avoided. To prevent the list of triangles from expanding too quickly due to addition and deletion, one of the three triangles in a deleted tetrahedron is replaced with the single replacement triangle, placing the other two on the dummy list. This is the inverse of the oneto-three addition move in which the original single triangle becomes one of the triangles of the new tetrahedron.

When a tetrahedron is deleted, three triangles are replaced with one. The triangle with the lowest index is retained [in Fig. 13, let this be triangle a (right)]. This triangle replaces the base of the tetrahedron, and so after deletion it becomes triangle $\mathbf{x}$ in Fig. 13 (left). Only the coordinates of the apex of this triangle are updated since it rotates about its base (in the case of Fig. 13, the 1-2 edge). The vertices involved may be defined intrinsically, without reference to their embedding space coordinates. The vertex that must be updated is the intersection of the vertices of all three triangles. The new vertex location is the vertex shared by the two triangles that are not the replacement triangle and which is not the apex. In Fig. 13, that is the vertex shared by triangles $\mathbf{b}$ and $\mathbf{c}$, but not shared by triangle $\mathbf{a}$. The coordinates of $\mathbf{a}^{0}$ are replaced with the coordinates of $\mathbf{b}^{0}$ or vertex $\mathbf{c}^{0}$ in the notation of Fig. 13.

The algorithm that performs deletion of tetrahedra depends on the fact that triangles can only be rotated in the surface; they cannot be flipped. It is convenient to define deletion in terms of an involution called inversion. When a vector is inverted, the vector is mapped to the other vector that occupies the same edge. Referring to Fig. 2, the pairs are
TABLE I. The sequence of relabelings that occur when the tetrahedron shown in Fig. 14 is replaced in a three-to-one move with the triangle a. $P$ indicates propagation and $I$ indicates inversion.

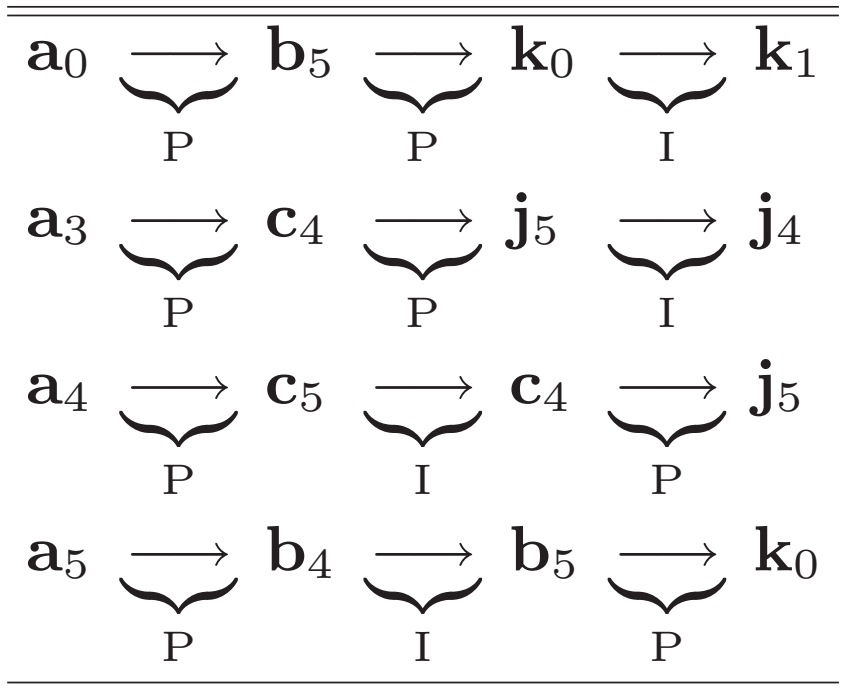

vectors $(0,1),(2,3)$, and $(4,5)$. If an inversion is performed on vector 3 , we get vector 2 , and so on. This involution is used, together with propagation along the vectors, to redefine the connectivity during deletion using only the intrinsic geometry information

For example, Fig. 14 shows a tetrahedron that will undergo deletion and be replaced with triangle $\mathbf{a}$. The connectivity for vectors $\mathbf{a}_{5}, \mathbf{a}_{0}, \mathbf{a}_{4}$, and $\mathbf{a}_{3}$ (where $\mathbf{a}_{i}$ is for vector $i$ of triangle a) must be redefined, as they point to triangles $\mathbf{b}$ and c which will be deleted. After deletion, when the tetrahedron is replaced with triangle $\mathbf{a}$, a particle occupying $\mathbf{a}_{5}$ will propagate to $\mathbf{k}_{0}$ if undisturbed by collision. Consider the propagation of a fictitious particle from $\mathbf{a}_{5}$ to $\mathbf{b}_{4}$. After a second propagation this particle would end up on triangle $\mathbf{c}$, which is incorrect. Inverting the position of the particle, so that it now occupies $\mathbf{b}_{5}$, and allowing the particle to propagate once more takes it to $\mathbf{k}_{0}$, which is correct. This was achieved by propagating once, inverting, and propagating again. The full set of relabelings given in terms of propagation and inversion is shown in Table I. Vectors $\mathbf{a}_{4}$ and $\mathbf{a}_{5}$ are readily identified as the vectors attached to the base of the lowest indexed triangle of the tetrahedron. They are updated to point to $\mathbf{j}_{5}$ and $\mathbf{k}_{0}$, respectively, as shown in Table I. The other two vectors may be updated by a similar sequence of propagation and inversion, but it is more straightforward to note that $\mathbf{a}_{0}$ is updated to $\mathbf{k}_{1}$, which is the inversion of $\mathbf{k}_{0}$, and $\mathbf{a}_{3}$ is updated to $\mathbf{j}_{4}$, which is the inversion of $\mathbf{j}_{5}$. Hence, the update of vectors $\mathbf{a}_{0}$ and $\mathbf{a}_{3}$ is obtained by inverting the images of $\mathbf{a}_{5}$ and $\mathbf{a}_{4}$, respectively.

A second useful involution is reflection, which returns the vector with opposite velocity on the triangle. A reflection on vector 1 , for example, returns vector 4 . Because deletion is allowed when spectator particles are present on the six edges of the replacement triangle, it is necessary to update the particle occupancy of the replacement triangle. It is straightforward, in terms of the involutions inversion and reflection, to identify the vectors whose particle occupancy needs to be translated to the replacement triangle. 


\section{Preventing degenerate triangulations}

In a combinatorial triangulation each triangle is uniquely defined by a set of three vertices: it is combinatorially unique. Triangulations which do not satisfy this criterion are degenerate. For example, in a degenerate triangulation two vertices may be connected by more than one edge or triangles may share more than one edge. As explained below, unrestricted application of the Pachner moves can result in degenerate triangulations.

One form of degeneracy occurs when two of a triangle's neighbors are the same triangle; in other words, two triangles share two edges, or only two triangles meet at a vertex. Such a feature resembles a "flap" attached to the rest of the triangulation. This type of degeneracy is avoided by preventing geometry moves whose postimage contains a flap. We now consider the effect of the two-to-two, one-to-three, and threeto-one moves from the point of view of avoiding degenerate triangulations.

First, a flap may be created by the two-to-two Pachner move. The two-to-two move increases or decreases the number of triangles at a vertex by one. If three triangles intersect at a point (the apex of a tetrahedron), this will become a flap if two of the triangles are replaced with one. The application of the two-to-two move to two of the triangles of a tetrahedron will therefore result in a flap. Preventing two triangles that are part of the same tetrahedron from undergoing a twoto-two move avoids this.

Second, a degenerate triangulation may not be produced by the one-to-three move (creation). Provided the initial triangulation is not degenerate, any vertex that is not at a boundary is shared by at least three triangles. The one-tothree move increases the number of triangles at each existing vertex by at least one, so this move can only create a flap if there were zero triangles to begin with. Hence, it is not possible for creation (the one-to-three move) to result in a degenerate triangulation

Third, it is possible for the three-to-one move (deletion) to produce a flap. The three-to-one move reduces the number of triangles at a vertex by one. This move can create a flap if the geometry as a whole is a tetrahedron, or if a tetrahedron is attached to a manifold by one edge. For example, a tetrahedron could be attached to the rest of the manifold via a "neck." To make this explicit, take a tetrahedron and label its faces $w x y z$. Let all the faces be connected except for $x$ and $y$. Now, take two adjacent triangles, $a$ and $b$, in the manifold that are not connected to each other, and glue the loose edge of $y$ to triangle $a$ and glue the loose edge of $z$ to triangle $b$. A triangulation with a feature like this is degenerate because the two vertices at the join between the tetrahedra and the rest of the triangulation are connected by two edges. If the tetrahedron that is attached by one edge of two triangles were to undergo a deletion of three of its faces, a flap would be created. This is illustrated in Fig. 15.

This type of structure can be produced from a tetraspiral geometry-the triangulation which results from successive reflections of each vertex of a tetrahedron through the opposing face [41]. In this geometry, and any geometry composed of tetrahedra sharing faces, a single three-to-one move results in two tetrahedra connected by a single edge. A second three-to-one move will then result in a flap.
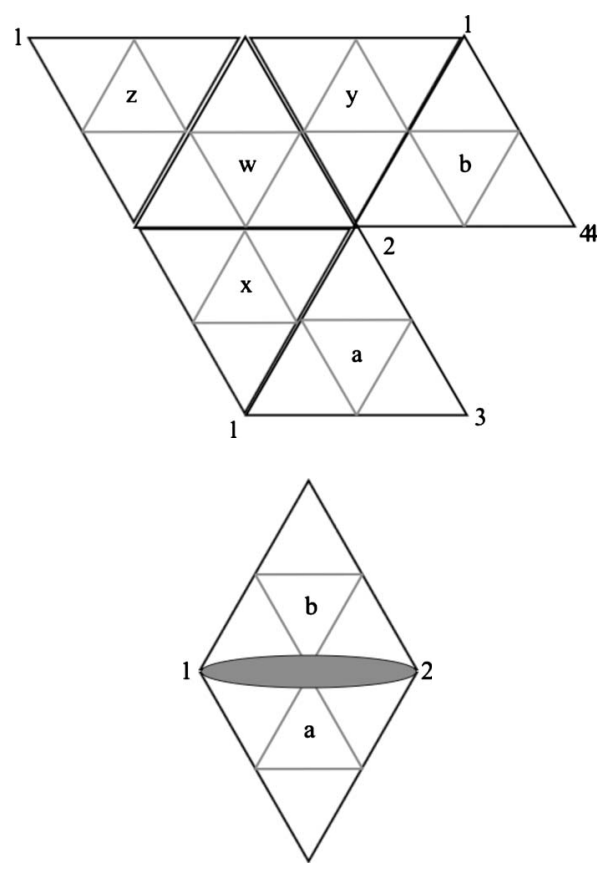

FIG. 15. A tetrahedron attached by an edge to the main body of the manifold. If deletion occurs to any triplet of the triangles $w-x-y-z$, a flap will be created.

Such degenerate triangulations are prevented from forming by two checks. The first one prevents degeneracy caused by two-to-two moves by checking prior to a two-to-two move that both triangles involved do not belong to the same tetrahedron. This prevents the formation of flaps and of tetrahedra attached by a shared edge. Second, we check that the three-to-one move is only applied to tetrahedra which are attached to the rest of the triangulation by three edges. It is straightforward to verify that flaps are not produced in our simulations by verifying, for example, that the vertex degree of all triangles is bounded below by three.

How does the prevention of such degenerate triangulations affect the time reversibility of the model? Consider the inversion of a sequence of moves involving the creation of a degenerate triangulation. Then disallowing the formation of degenerate triangulations corresponds to disallowing degenerate triangulations in the preimage of the inverse rule. If such triangulations are not allowed to form, one must naturally forbid them in the initial geometry. Disallowing degenerate triangulations in the initial condition is sufficient to maintain reversibility. If such triangulations are allowed in the initial data, but not in the dynamics, invertibility is violated because states exist with two preimages: e.g., one where a flap has been removed due to addition and one where it was prevented from forming.

\section{GEOMETRY DYNAMICS}

In this section we study some aspects of the dynamics of the geometry degrees of freedom. In these simulations the fluid represented by the particles is quiescent-there is no forcing applied, and because the initial velocities of the particles are assigned randomly the average hydrodynamic ve- 
locity field will be zero. In one dimension, where the only geometrical degree of freedom is the size of the lattice, both numerical simulation and calculations for particular sets of initial conditions result in an average growth of the lattice size of $t^{1 / 2}$ [33-35]. We perform the comparable calculations and mean-field theory treatment of the two-dimensional model. In addition, because the varying vertex degree of the triangulation represents a local geometrical degree of freedom, we also present preliminary results on the distribution of vertex degree on the manifold.

\section{A. Mean-field theory}

In this section we consider the average behavior of the number of triangles in the model as a function of time. First note that deletion is a rare event compared to addition. Addition requires exactly three particles in one of the two configurations of a single triangle. Deletion requires exactly three particles in one of the two configurations of three triangles. If all configurations of a triangle occur with equal probability, deletion will be less likely than addition simply because it requires correlations between the states of more than one triangle. Since addition is more common than deletion, both by this argument and by observation of actual simulations, we construct a mean-field prediction for the behavior of our system with only addition of geometry. Meanfield predictions tend to fail for low-dimensional systems. In the one-dimensional case, for example, the lattice grew as $t^{1 / 2}$, but a mean-field model predicted $t^{1 / 3}$ [34]. It is therefore of interest to determine the validity of the mean-field prediction in two dimensions.

Let $N$ represent the number of particles and $S$ represent the number of triangles. The mean number of particles per site is given by

$$
\rho=\frac{N}{S}, \quad 0 \leq \rho \leq 6 .
$$

The probability for a site to undergo addition, $P_{+}$, will be proportional to the probability that three sites are occupied and three sites are unoccupied,

$$
P_{+} \propto \rho^{3}(1-\rho)^{3} .
$$

The expected number of triangles which will undergo addition is

$$
\left\langle S_{+}\right\rangle=S P_{+} \propto S \rho^{3}(1-\rho)^{3} .
$$

The expected change in the number of triangles is given by

$$
\begin{gathered}
\Delta S=2\left\langle S_{+}\right\rangle \propto S \rho^{3}(1-\rho)^{3}, \\
\Delta S \propto\left(\frac{N^{3}}{S^{2}}-3 \frac{N^{4}}{S^{3}}+3 \frac{N^{5}}{S^{4}}+\frac{N^{6}}{S^{5}}\right) .
\end{gathered}
$$

In the limit in which creation dominates deletion and the number of particles, $N$, is conserved, the first term in the equation above will dominate. Disregarding the last three terms, which will become small as the number of triangles, $S$, grows, we convert this to a differential equation and solve:

$$
\begin{aligned}
& \frac{d S}{d t} \propto \frac{N^{3}}{S^{2}}, \\
& S \propto t^{1 / 3} .
\end{aligned}
$$

The mean-field prediction is therefore that the lattice will grow asymptotically as $t^{1 / 3}$.

\section{B. Results}

Four different types of simulations were performed, each without restrictions upon the curvature of the manifold (except those arising from forbidding degenerate triangulations) or the embedding. All simulations began from an initial icosahedral geometry in which each triangular face was subdivided into 16 triangles by repeatedly bisecting the edges. Simulations were performed with only creation; only creation and deletion; only creation and the two-to-two rule; and a simulation with creation, deletion, and the two-to-two rule. While simulations with creation but no deletion are not time reversible, they test the hypothesis that the geometry dynamics is dominated by addition. 30 realizations were performed for each type of simulation with $10^{5}$ time steps each to determine the number of triangles as a function of time. The data were fitted to a power law, $S(t)=a t^{b}$, where $S(t)$ represents the number of triangles in the lattice and $t$ is the number of time steps. Fitting the data to the form $S(t)=a\left(t-t_{0}\right)^{b}$ gave values of $t_{0}$ of order 1 , showing that there is only a short transient before the power-law growth begins, and so fitting the data to $S(t)=a t^{b}$ is appropriate.

To evaluate the fit $\chi^{2}$ per degree of freedom for the fit function $L(t)=a t^{b}$ was computed:

$$
\chi^{2}(a, b)=\frac{1}{n-p} \sum_{i} \frac{\left[\left\langle S\left(t_{i}\right)\right\rangle-f\left(t_{i}, a, b\right)\right]^{2}}{\sigma_{i}^{2}},
$$

where $n$ is the number of data points; $p$ is the number of parameters, in this case $2, a$ and $b$; and $\sigma_{i}$ is the standard deviation on the mean for each $\left\langle S_{i}\right\rangle$, where

$$
\left\langle S_{i}\right\rangle=\frac{1}{r} \sum_{j} S_{i j},
$$

and $j$ is the number of realizations.

For each type of simulation an initial fit using ORIGIN 7.0 [42] was obtained (using a Levenberg-Marquardt method) and an independent error analysis was performed by computing $\chi^{2}$ in the $a b$ plane. The minimum value of $\chi^{2}$ found via this method matches that found by ORIGIN 7.0. The parameter uncertainties were obtained by this $\chi^{2}$ analysis by allowing $\chi^{2}$ to increase by one above the minimum. The uncertainties so obtained are larger than those given by ORIGIN, presumably because we allow $a$ and $b$ to vary independently. In all four types of simulation the exponent value is consistent with a power-law exponent of $1 / 3$, in agreement with the meanfield prediction. The data and $\chi^{2}$ analysis for the simulations with all Pachner moves are shown in Fig. 16 and those for the simulations with only the three-to-one (addition) Pachner move are shown in Fig. 17, with all Pachner moves except the three-to-one move (deletion) in Fig. 18 and with all Pachner moves except the two-to-two move in Fig. 19. 

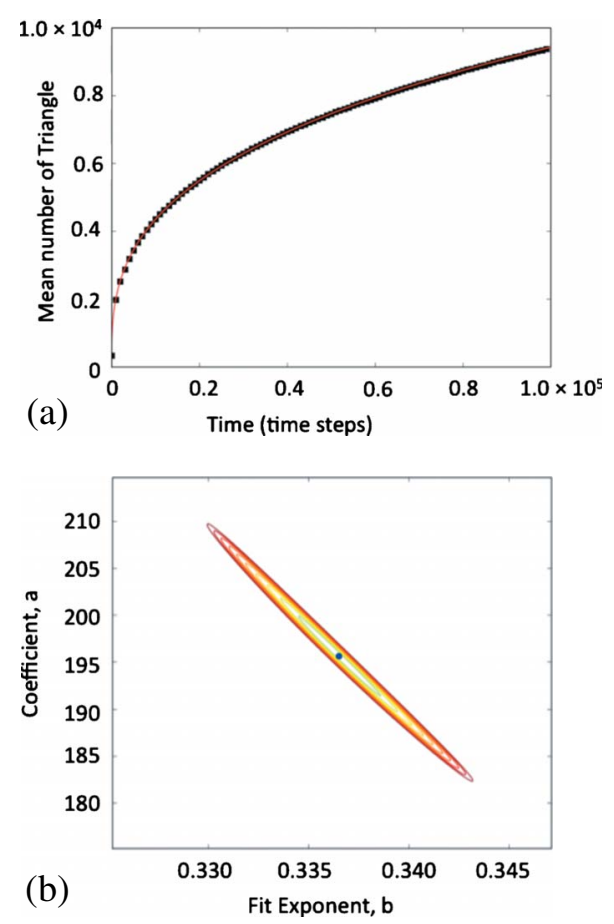

FIG. 16. (Color online) All Pachner moves allowed. Number of triangles as a function of time averaged over 30 realizations for 100000 time steps each with no restrictions on the curvature or embeddability of the triangulation, and all Pachner moves utilized. The symbols in (a) show the simulation data every 1000 time steps and are larger than one standard deviation of the mean. The solid line is a fit created in ORIGIN 7.0 with a Levenberg-Marquardt method for $L(t)=a t^{b}$ to the complete data set of 100000 points. (b) shows a contour plot of $\chi^{2}$ using a sampling of 200 points evenly spaced along the range of $a$ and $b$. The minimum $\chi^{2}$ value lies at $\chi^{2}=0.0319$ at $a=196$ and $b=0.33618$ in agreement with the fit found by ORIGIN 7.0, and the outermost contour represents a deviation of 1.0 from this minimum. The fitted value of the exponent is $b=0.33618 \pm 0.0065$, consistent with a power-law exponent of $1 / 3$.

\section{Curvature analysis}

Unlike the one-dimensional model it is possible to define a curvature variable at each vertex of the triangulation. As we do not restrict our triangulations in any way in the simulations described above, it is of interest to quantify how curvature is distributed for a typical realization. We performed four simulations of a single realization of the type of simulation displayed in Figs. 16-19. A histogram of the vertex degree is shown in Fig. 20. As expected, by allowing unrestricted addition of tetrahedron vertices of arbitrarily high degrees form in the triangulation. However, most of the vertices of the triangulation have degrees between 3 and 10 . While the data shown in Fig. 20 are insufficient to support a detailed quantitative analysis of the distribution of vertex degree, it appears by inspection to be consistent with an exponential distribution for degrees between 3 and 10. Larger vertex degrees appear to be more common than that predicted by this trend below vertex degree 10, but there are insufficient data to draw conclusions here. If we denote the number of tetrahedra added to the original geometry $N_{1}$, and tetrahedra added to these $N_{2}$ and so on, an exponential dis-
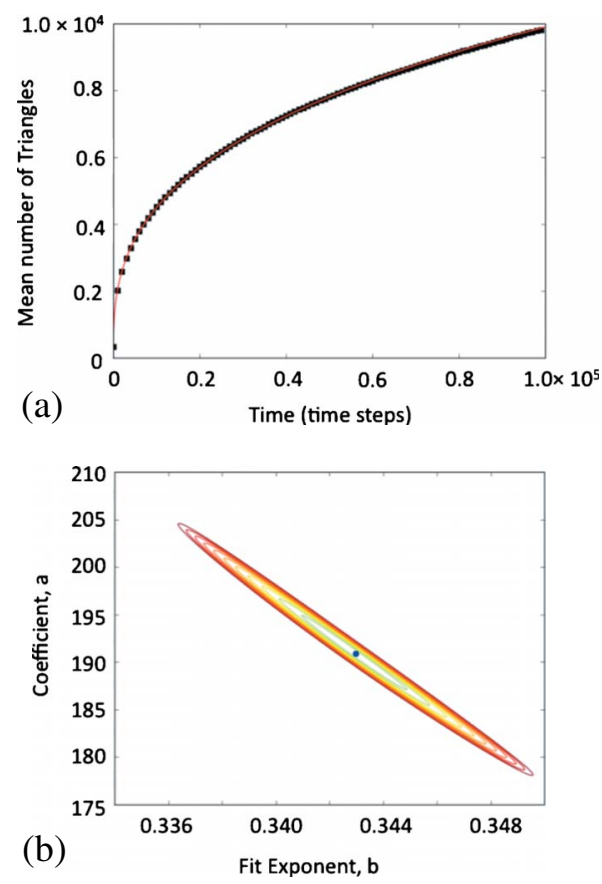

FIG. 17. (Color online) Only addition of tetrahedra. Number of triangles as a function of time averaged over 30 realizations for 100000 time steps each. The simulations included only the one-tothree Pachner move with no growth or embedding control. The symbols in (a) show the simulation data every 1000 time steps and are larger than one standard deviation of the mean. The solid line is a fit created in ORIGIN 7.0 with a Levenberg-Marquardt method for $L(t)=a t^{b}$ to the complete data set of 100000 points. (b) shows a contour plot of $\chi^{2}$ using a sampling of 200 points evenly spaced along the range of $a$ and $b$. The minimum $\chi^{2}$ value lies at $\chi^{2}$ $=1.106$ at $a=190.864$ and $b=0.343$ in agreement with the fit found by ORIGIN 7.0, and the outermost contour represents a deviation of 1.0 from this minimum. The fitted value of the exponent is 0.337 $\leq b=0.344 \leq 0.350$, consistent with a power-law exponent of $1 / 3$.

tribution is consistent with the ratio of $N_{i}$ to $N_{i+1}$, being a constant.

In Fig. 21 we display visualizations of the triangulation for a typical realization. This figure shows a simulation in which only the three-to-one Pachner move is implemented, resulting in a triangulation which is always isometrically immersible in three dimensions. This simulation shows that these triangulations self-intersect many times. This results in the appearance of many small triangles in the visualization-in fact these are parts of triangles which intersect each other. Visualizations of realizations with any combination of rules applied all showed this feature. In addition, one observes clusters of added tetrahedra in all simulations. This may be due to the fact that added tetrahedra act as scattering centers for particles, and so groupings of tetrahedra will naturally increase the probability of further geometry-changing collisions by causing particles to spend longer in a given region.

\section{CONCLUSION}

We have presented a lattice gas model with dynamical geometry in two dimensions. Our model is an extension of 

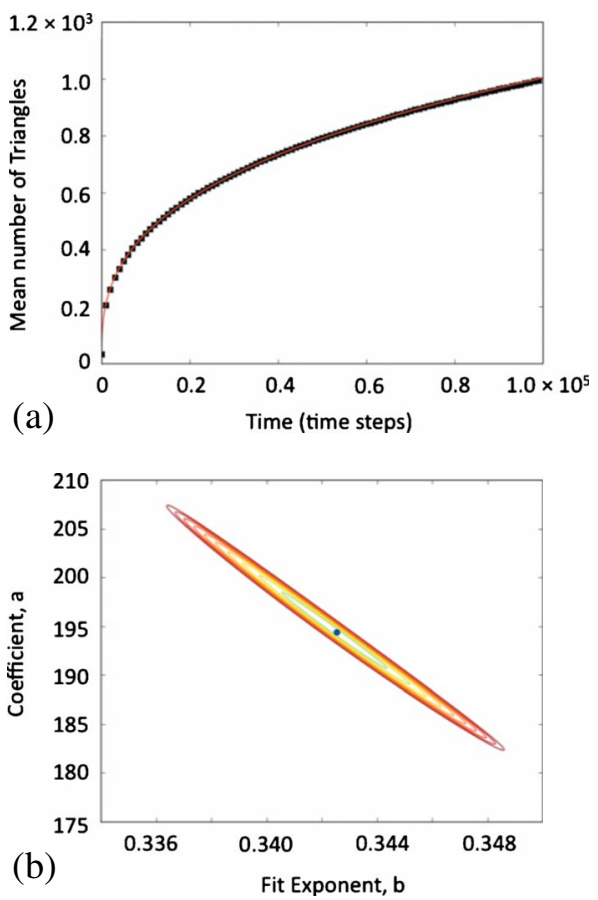

FIG. 18. (Color online) No deletion. Number of triangles as a function of time averaged over 30 realizations for 100000 time steps each. The simulations included both two-to-two and one-tothree Pachner moves with no growth or embedding control. The symbols in (a) show the simulation data every 1000 time steps and are larger than one standard deviation of the mean. The solid line is a fit of $L(t)=a t^{b}$ to the complete data set of 100000 points. The fit was created in ORIGIN 7.0 using a Levenberg-Marquardt method. (b) shows a contour plot of $\chi^{2}$ using a sampling of 200 points evenly spaced along the range of $a$ and $b$. The minimum $\chi^{2}$ value lies at $\chi^{2}=0.878$ at $a=194.402$ and $b=0.343$ in agreement with the fit found by ORIGIN 7.0. The outermost contour represents a deviation of 1.0 from this minimum. The fitted value of the exponent is $0.336 \leq b=0.343 \leq 0.349$, consistent with a power-law exponent of $1 / 3$.

the FHP hydrodynamic two-dimensional lattice gas model and the one-dimensional dynamical geometry lattice gas [3,33-35]. We have defined and implemented rules for dynamical geometry by both Pachner moves. For a quiescent fluid on an initially icosahedral geometry the number of triangles grows as $t^{1 / 3}$ for all combinations of rules simulated. This is in agreement with a mean-field prediction, a fact of some interest as mean-field predictions generally fail in low dimensions and in fact fails for the one-dimensional version of this model $[34,35]$.

Unlike the one-dimensional case, the flat space limit of this model is nontrivial: it is the hydrodynamic FHP lattice gas. For our model as defined it is therefore possible to perform simulations in three regimes. First, the fluid may be quiescent, and the geometry dynamical, the limit studied in this paper. Second, the geometry may be fixed and nontrivial, and the fluid driven. This regime is relevant for the simulation of surface flows on fixed background geometry, such as atmospheric flows on the sphere, and in experiments with curved soap films [8]. Third, the fluid may be driven and the geometry dynamical, a situation of relevance to surface flows
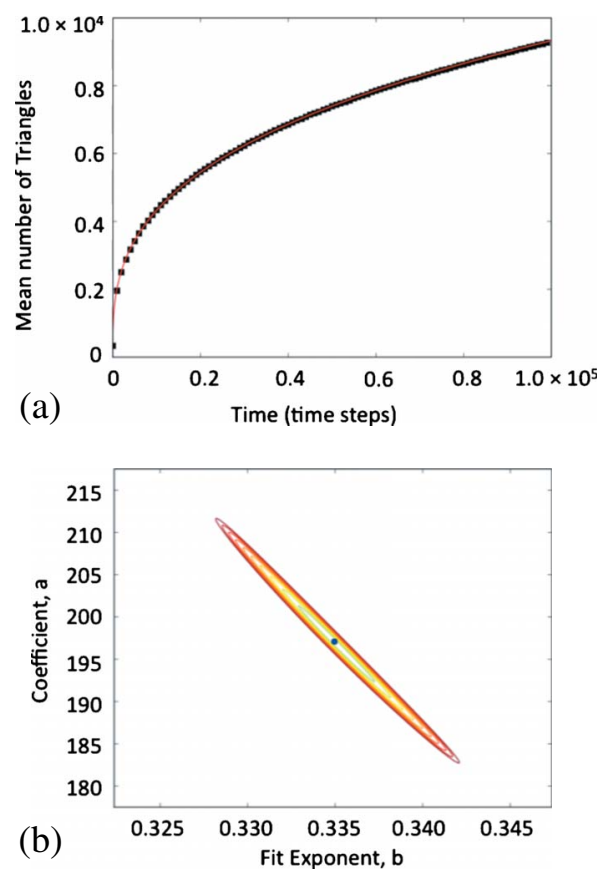

FIG. 19. (Color online) No two-to-two moves allowed. Number of triangles as a function of time averaged over 30 realizations for 100000 time steps each. The simulations included both the one-tothree and three-to-one Pachner moves with no two-to-two moves and without restrictions on the curvature or embeddability of the triangulation. The symbols in (a) show the simulation data every 1000 time steps and are larger than one standard deviation of the mean. The solid line is a fit of $L(t)=a t^{b}$ to the complete data set of 100000 points. The fit was created in ORIGIN 7.0 using a LevenbergMarquardt method. (b) shows a contour plot of $\chi^{2}$ using a sampling of 200 points evenly spaced along the range of $a$ and $b$. The minimum $\chi^{2}$ value lies at $\chi^{2}=0.666$ at $a=197.060$ and $b=0.335$ in agreement with the fit found by ORIGIN 7.0. The outermost contour represents a deviation of 1.0 from this minimum. The fitted value of the exponent is $0.330 \leq b=0.335 \leq 0.342$, consistent with a powerlaw exponent of $1 / 3$.

in fluid interfaces. Indeed, the equations for surface flow are well known, including the case in which the surface is dynamical [22,23].

Given the model defined here, a natural question to pose is what are the macrodynamical equations of motion? In the regime where a nontrivial flow occurs on a fixed background geometry, are the relevant fluid equations the Navier-Stokes equations on the manifold represented by the triangulation? In the regime where the geometry is dynamical, does the time evolution of the flow coupled to the geometry obey the continuum equations of surface flow given in $[22,23]$ ?

The principal tool used to obtain the macrodynamical equations of a given lattice gas model is the ChapmanEnskog expansion [43]. This is an asymptotic expansion around a local equilibrium distribution. It is valid in the regime that local equilibrium, characterized by a few hydrodynamic fields, is reached rapidly, while global equilibration occurs on longer time scales by hydrodynamic processes. Analysis of the model defined in this paper requires a new variation of the Chapman-Enskog expansion. 


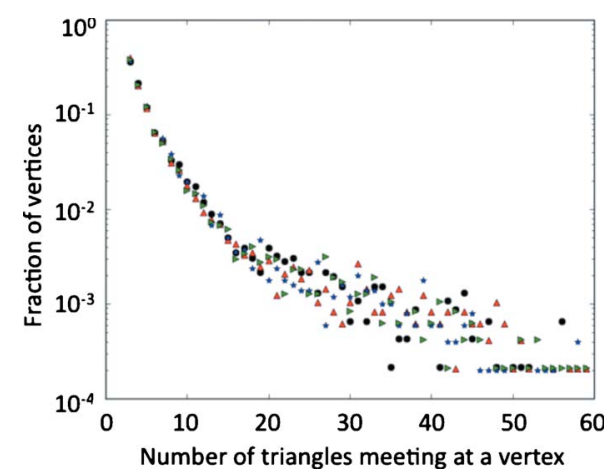

FIG. 20. (Color online) Histogram of number of triangles meeting at each vertex. Results of four separate simulations for 100000 time steps. The initial geometry was an icosahedron with each of its faces subdivided into 16 equilateral triangles. Data are shown for one simulation with all Pachner moves implemented (black circles), one simulation with only the three-to-one addition move implemented (red upward-pointing triangles), one simulation with the one-to-three addition move and the two-to-two move but no deletion (blue stars), and one simulation with addition and deletion moves but no two-to-two move (green sideway-pointing triangles).

To treat the model on a fixed curved surface the Chapman-Enskog analysis would need to be extended to arbitrary two-dimensional manifolds, work which will be presented in a separate paper [40]. To treat the case where the geometry becomes dynamical, it must be possible to introduce the geometry degrees of freedom into the ChapmanEnskog analysis. One way to do this is to define the continuum limit of the triangulation in the same way as the continuum limit of the velocity field. That is, one considers an average (time, spatial, or ensemble) over many triangulations of the same surface. The macrodynamical equations of surface flow given in [22,23] might then arise, for suitably chosen collision rules, from a Chapman-Enskog analysis as the slow relaxation of fluid plus geometry after a fast relaxation to an equilibrium geometry. If such an analysis is valid for the model defined in this paper, it would also allow simulation of fixed geometries via simulation of an ensemble of dynamical geometries fluctuating about an average continuum surface.

The equilibrium statistical mechanics of two-dimensional triangulated surfaces embedded in three dimensions has been well studied [16-18]. The model defined here differs from this body of work in several ways. The tethered surfaces studied in [16] have a fixed internal metric and a Hamiltonian which depends only on extrinsic embedding coordinates. The triangulations of our model have an intrinsic metric which varies dynamically due to application of the Pachner moves. In the terminology of [16] this makes our surfaces liquid rather than tethered. The object of study of [16] and subsequent work was the equilibrium properties of embedded surfaces; here we are interested in the nonequilibrium dynamics of surfaces on which there is a nontrivial vector field whose dynamics is coupled to the intrinsic geometry of the triangulation.

However, for the case simulated in this paper in which the fluid degrees of freedom are quiescent it is interesting to compare the typical geometries shown in Fig. 21 with the
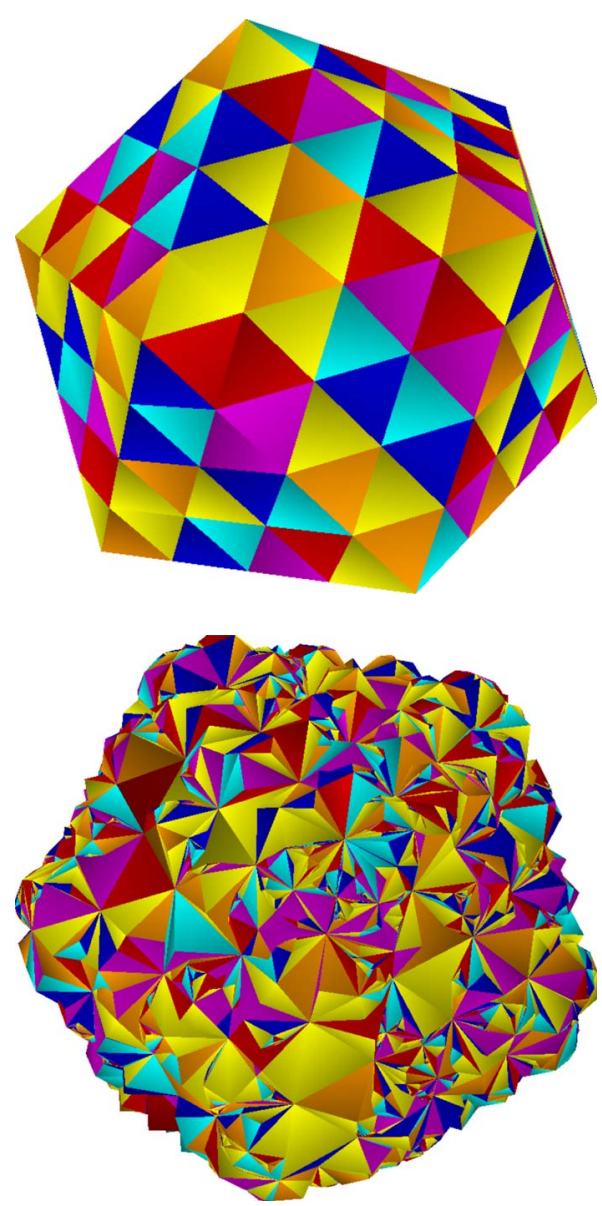

FIG. 21. (Color online) Visualizations of the triangulation for a typical realization. (a) Initial condition for all simulations. (b) Typical geometry after 100000 time steps for a simulation with only the three-to-one Pachner move (addition) implemented, with no restriction on the curvature. The faces shown here are individual triangles - with only the three-to-one Pachner move implemented the immersion being always isometric. The appearance of many smaller triangles is due to the fact that the surface is extensively self-intersected.

equilibrium geometries in the crumpled phase of random surface models. Two observations are relevant. First, as we allow self-intersection and do not restrict the embedding, our surfaces are phantom surfaces and should be compared with random surface models which have no extrinsic curvature terms in their Hamiltonians. The equilibrium geometries of such random surface models are crumpled and contain many "spiky" features.

The typical geometries in our simulations exhibit similar features - the high-degree vertices shown in Fig. 20 occur at branching points where many tetrahedra share a common vertex. The geometries shown in Fig. 21 also exhibit a concentration of new tetrahedra-showing that tetrahedra are added on new tetrahedra more often than on the original geometry. This can be explained by the fact that the curvature produced by new geometry will act as scattering centers for the particles-causing particles to spend longer in the vicinity of new geometry, where they will then scatter and add further new geometry. This will naturally lead to a 
branched polymerlike structure where tetrahedra are added to tetrahedra, and particles become trapped on the new branches of the geometry.

Future study of the model should determine whether the geometries produced by the model without constraint are indeed in the crumpled phase. Simulations in which constraints are applied to the local curvature or embedding of the triangulation may result in geometries closer to smooth manifolds and so may be necessary for applications in which one aims to simulate a fluid moving on a smooth two-dimensional surface. The most natural area of application of the proposed model is in the simulation of fluid interfaces in which the interface is changing dynamically. The situation of fixed geometry is an intermediate situation of interest primarily for characterizing the model.

Beyond the practical applications of extending lattice gas methods to situations of dynamical geometry, the proposed model also provides a simple conceptual arena for consideration of issues which arise beyond fluid mechanics. Fluids, while frequently treated as continua, are in fact composed of atoms or molecules. The lattice gas and lattice-Boltzmann methods use the existence of an underlying statistical description of a fluid to realize efficient numerical methods for fluid simulation $[5,6]$. While a discretization of space and time underpins most numerical methods for field theories, our most fundamental current understanding is that spacetime is a continuous Lorentzian manifold.

The idea that, like fluids, spacetime may have underlying discrete substructure occurs repeatedly in speculative models for quantum gravity. A treatment of classical general relativity on polyhedral simplicial complexes was first considered by Regge [12]. In the causal dynamical triangulations approach the four space-time dimensions emerge from an ensemble of simplicial complexes, suitably constrained by causality [44]. In the causal sets approach the underlying Lorentzian manifold is replaced with a discrete set of points with a causal (partial) order [45]. In loop quantum gravity geometrical operators such as area and length have a discrete spectrum [46]. In discrete models of quantum gravity the apparently continuous classical spacetime emerges at large scales due to the smallness of the Planck length. In the more experimentally accessible world of fluid dynamics, the continuum picture is valid because of the largeness of Avogadro's number. The discrete model of fluid mechanics on arbitrary triangulated surfaces presented here provides a model in which the question of the emergence of smooth manifoldlike structures, and associated dynamics of a classical field on the manifold, may be studied without the numerous conceptual problems of both general relativity and quantum mechanics.

\section{ACKNOWLEDGMENTS}

This work received financial support from Research Corporation for Science Advancement through a Cottrell College Science Award, and from the Sherman Fairchild Foundation and Howard Hughes Medical Institute. P.J.L. thanks Bruce Boghosian, Gianluca Caterina, Suzanne Amador-Kane, and Stephon Alexander for stimulating discussions, and the Department of Mathematics at UCSD and the Institute for Quantum Information at Caltech for hosting visits during which parts of this work were completed.
[1] L. P. Kadanoff and J. Swift, Phys. Rev. 165, 310 (1968).

[2] J. Hardy, O. de Pazzis, and Y. Pomeau, Phys. Rev. A 13, 1949 (1976).

[3] U. Frisch, B. Hasslacher, and Y. Pomeau, Phys. Rev. Lett. 56, 1505 (1986).

[4] S. Wolfram, J. Stat. Phys. 45, 471 (1986).

[5] G. D. Doolen, Lattice Gas Methods for Partial Differential Equations (Addison-Wesley Publishing Company, Reading, MA, 1990).

[6] S. Succi, The Lattice-Boltzmann Equation for Fluid Dynamics and Beyond (Oxford University Press, Oxford, 2001).

[7] J. A. Dutton, The Ceaseless Wind: An Introduction to the Theory of Atmospheric Motion (McGraw Hill, New York, 1986).

[8] F. Seychelles, Y. Amarouchene, M. Bessafi, and H. Kellay, Phys. Rev. Lett. 100, 144501 (2008).

[9] G. Gompper and M. Schick, Phase Transitions and Critical Phenomena, edited by C. Domb and J. Lebowitz (Academic, London, 1994), Vol. 16, pp. 1-181.

[10] U. Pachner, Eur. J. Comb. 12, 129 (1991).

[11] A. Einstein, Ann. Phys. (Leipzig) 49, 769 (1915).

[12] T. Regge, Nuovo Cimento 19, 558 (1961).

[13] J. Ambjorn, Z. Burda, J. Jurkiewicz, and C. Kristjansen, Acta Phys. Pol. B 23, 991 (1992).
[14] R. Loll, Class. Quantum Grav. 25, 114006 (2008).

[15] M. Kardar, G. Parisi, and Y. C. Zhang, Phys. Rev. Lett. 56, 889 (1986).

[16] Y. Kantor, M. Kardar, and D. R. Nelson, Phys. Rev. Lett. 57, 791 (1986).

[17] Y. Kantor, M. Kardar, and D. R. Nelson, Phys. Rev. A 35, 3056 (1987).

[18] J. F. Wheater, J. Phys. A 27, 3323 (1994).

[19] M. Paczuski, M. Kardar, and D. R. Nelson, Phys. Rev. Lett. 60, 2638 (1988).

[20] C. Marangoni, Nuovo Cimento Ser. 2 7-8, 301 (1872).

[21] P. J. Love and P. V. Coveney, Philos. Trans. R. Soc. London, Ser. A 360, 357 (2002).

[22] L. E. Scriven, Chem. Eng. Sci. 12, 98 (1960).

[23] R. Aris, Vectors, Tensors, and the Basic Equations of Fluid Mechanics (Dover, New York, 1962).

[24] B. Widom, J. Chem. Phys. 84, 6943 (1986).

[25] B. M. Boghosian, P. V. Coveney, and A. N. Emerton, Proc. R. Soc. London, Ser. A 452, 1221 (1996).

[26] P. V. Coveney, A. N. Emerton, and B. M. Boghosian, J. Am. Chem. Soc. 118, 10719 (1996).

[27] A. N. Emerton, P. V. Coveney, and B. M. Boghosian, Physica A 239, 373 (1997).

[28] A. N. Emerton, P. V. Coveney, and B. M. Boghosian, Phys. 
Rev. E 56, 1286 (1997).

[29] A. N. Emerton, F. W. J. Weig, P. V. Coveney, and B. M. Boghosian, J. Phys.: Condens. Matter 9, 8893 (1997).

[30] B. M. Boghosian, P. V. Coveney, and P. J. Love, Proc. R. Soc. London, Ser. A 456, 1431 (2000).

[31] P. J. Love, P. V. Coveney, and B. M. Boghosian, Phys. Rev. E 64, 021503 (2001).

[32] P. J. Love, J.-B. Maillet, and P. V. Coveney, Phys. Rev. E 64, 061302 (2001).

[33] B. Hasslacher and D. Meyer, Int. J. Mod. Phys. C 9, 1597 (1998).

[34] P. J. Love, B. M. Boghosian, and D. A. Meyer, Philos. Trans. R. Soc. London, Ser. A 362, 1667 (2004).

[35] K. Baur, J. Rabin, and D. Meyer, Phys. Rev. E 73, 026129 (2006).

[36] P. D. D. Scherer and B. Sherwood, Comput. Sci. Eng. 2, 56 (2000).

[37] B. M. Boghosian, P. J. Love, and D. A. Meyer, Philos. Trans.
R. Soc. London, Ser. A 360, 333 (2002).

[38] An immersion is an embedding in which self-intersection of the surface is allowed.

[39] J. Nash, Ann. Math. 63, 20 (1956).

[40] D. Cianci and P. J. Love (unpublished).

[41] C. Zheng, R. Hoffmann, and D. Nelson, J. Am. Chem. Soc. 112, 3784 (1990).

[42] P. M. Edwards, J. Chem. Inf. Comput. Sci. 42, 1270 (2002).

[43] S. Chapman and T. Cowling, The Mathematical Theory of Non-Uniform Gases (Cambridge University Press, Cambridge, UK, 1939).

[44] J. Ambjorn, R. Loll, W. Westra, and S. Zohren, J. High Energy Phys. 12(2007), 017.

[45] L. Bombelli, J. Lee, D. Meyer, and R. D. Sorkin, Phys. Rev. Lett. 59, 521 (1987).

[46] C. Rovelli, Quantum Gravity (Cambridge University Press, Cambridge, UK, 2004). 